Original paper

\title{
Clinical effects of simvastatin in chronic hepatitis $C$ patients receiving sofosbuvir/daclatasvir combination. A randomized, placebo-controlled, double-blinded study
}

\author{
Hossam Z Mohamed', Nagwa A Sabri' ${ }^{2}$ Hossam M Zaki', Sara M Shaheen² \\ 'Hepatology Department, Kobri El koba Armed Forces Hospital, Cairo, Egypt \\ ${ }^{2}$ Department of Clinical Pharmacy, Faculty of Pharmacy, Ain Shams University, Cairo, Egypt
}

\begin{abstract}
Aim of the study: Chronic hepatitis C (CHC) affects more than 71 million people worldwide. Many therapies containing different direct-acting antivirals (DAAs) are now used. However, lipid profile is considered an important outcome with DAAs. So, this study aimed to assess clinical effects of statins in CHC patients.

Material and methods: One hundred patients were recruited from Kobri El koba Armed Forces Hospital and randomly assigned to: the drug group ( $D ; n=50)$ receiving simvastatin $10 \mathrm{mg}$ plus sofosbuvir $400 \mathrm{mg} /$ daclatasvir $60 \mathrm{mg}$ (SOF/DAC) daily for 12 weeks; and the placebo group ( $P ; n=50$ ), receiving placebo plus the same (SOF/DAC) regimen. Sustained virological response at 12 weeks after treatment (SVR12), lipid profile, C-reactive protein (CRP) and fibrosis stage were assessed.

Results: One hundred treatment-naiive $\mathrm{CHC}$ patients completed 12 weeks of the protocol with no clinically significant side effects. There was an increase in SVR failure rate in $P(10 \%)$ compared to $D$ (only $2 \%$ ) but not reaching statistical significant difference; SVR12 $(p>0.05)$. Logistic regression analysis showed that high baseline CRP, low baseline hemoglobin level and non-statin usage had an independent effect on increasing the probability of SVR failure in both groups; $p=0.03, p=0.0028, p=0.02$, respectively.

Conclusions: Statins could have an irreplaceable role in successful treatment of CHC patients receiving sofosbuvir/daclatasvir.
\end{abstract}

Key words: chronic hepatitis C, statins, sofosbuvir/daclatasvir.

\section{Address for correspondence}

Dr. Sara M Shaheen, Department of Clinical Pharmacy, Faculty of Pharmacy, Ain Shams University, Monazamet El-Wehda El-Afriqeya St., Abassia, Cairo, Egypt, 11566, e-mail: drsara61181@gmail.com

\section{Introduction}

Hepatitis C virus (HCV) is one of the most common causes of chronic liver disease and the leading indication for liver transplantation worldwide [1]. In Egypt, $\mathrm{HCV}$ infection is a major public health burden, where it bears the highest prevalence rate in the world [2].

HCV utilizes peripheral lipid metabolism pathways including hepatocyte very-low-density lipoprotein (VLDL) for viral assembly and requires several apolipoproteins for production of infective particles [3]. As a result, chronic hepatitis $\mathrm{C}(\mathrm{CHC})$ is associated with reduced total cholesterol, low-density lipoprotein (LDL) and apolipoprotein B (ApoB) levels as well as an increased rate of insulin resistance (IR) and type 2 diabetes mellitus [4].

On the other hand, total cholesterol, LDL cholesterol, and high-density lipoprotein cholesterol levels increased after therapy regardless of the regimen [5].

According to Lang et al., clinical studies have shown that chronic hepatitis $\mathrm{C}$ is associated with hypocholesterolemia and low levels of LDL. On the other hand high pretreatment cholesterol levels have been proposed to enhance the chance to achieve a sustained 
virologic response (SVR), although results of these studies were conflicting [6]. However, Hsu et al. reported that viral eradication due to IFN may significantly decrease cardiovascular (CV) morbidity [7]. But data regarding the effect of direct-acting antiviral (DAA) on glucose and lipid metabolism are incomplete, extrapolated from clinical trials and partially contradictory [4]. Recently, the findings demonstrated that DAA treatment may increase levels of triglyceride (TG), cholesterol and TG/cholesterol ratio loaded on a single VLDL particle in patients with chronic hepatitis C [8].

Meissner et al. reported that serum cholesterol level is increased soon after initiation of sofosbuvir plus ledipasvir (SOF + LDV) therapy [9]. Moreover, the clearance of HCV using combination therapy with daclatasvir (DAC) and asunaprevir (ASV), an IFN-free antiviral regimen, resulted in rapid changes in serum lipid profile and disturbed lipid metabolism, whereas results reported by Jain and his colleagues showed that total cholesterol levels and LDL levels were significantly elevated after SOF/DAC treatment, which may impact the cardiovascular events associated with hepatitis $\mathrm{C}$ treatment [10]. Statins are HMG-CoA reductase inhibitors which have been shown to play an important role in the modulation of hepatic steatosis, cholesterol metabolism and fibrosis. Moreover, Butt et al. showed that statin use was associated with improved antiviral efficacy as well as decreased progression of liver fibrosis and a reduced incidence of hepatocellular carcinoma (HCC) [11].

Statins appear to block HCV replication by inhibiting de novo cholesterol and geranylgeranylated protein synthesis, thus reducing expression of key HCV viral proteins and inhibiting pro-inflammatory signaling pathways $[12,13]$.

The role of statins as adjunctive therapy in HCV treatment has so far been limited to the previous standard of care, pegylated interferon and ribavirin. Furthermore, in vitro studies have shown that statins increase the antiviral activity of different DAAs in an additive manner and delay or even prevent the development of resistance against DAAs [14].

According to Sara et al., their search of the PubMed database and UMIN Clinical Trials Registry System, no clinical trial has been conducted for the combination of statins and DAAs [15]. This raised the need to determine the therapeutic benefits of statins in combination with new DAAs during treatment of $\mathrm{HCV}$ infected patients.

The current study was conducted to investigate the clinical benefits of using simvastatin in $\mathrm{CHC}$ patients receiving sofosbuvir/daclatasvir combination in terms of amelioration of lipid profile and glycemic status.

\section{Material and methods}

\section{Patients and study design}

This study was a prospective, interventional, randomized, placebo-controlled, double-blinded pilot study that included 100 adult (18-75 years old) male Egyptian patients with chronic HCV infection who were diagnosed by anti-HCV antibodies and HCV-RNA in addition to the histological evidence of chronic hepatitis. Patients were recruited from the outpatient clinic of Kobri El koba Armed Forces Hospital, Cairo, Egypt.

The study protocol was approved by the ethical committee of the Faculty of Pharmacy, Ain Shams University, Egypt, and has been registered on ClinicalTrials.gov with the identifier number NCT03490097. This study was conducted in accordance with the principles outlined in the Good Clinical Practice standard and the Declaration of Helsinki. Prior to participation, all patients were informed about the study protocol and they signed a written informed consent form.

\section{Patients eligibility}

Patients included in the study were those who fulfilled the inclusion criteria of the National Committee for Control of Viral Hepatitis, Ministry of Health and Population (MOHP), Egypt, and were male patients age 18 to 75 years old, in the easy-to-treat group: treatment-naïve patients with serum HCV RNA positivity by PCR, clinically stable condition outpatients, platelet count $\geq 150,000 / \mathrm{mm}^{3}$, patients with INR $\leq 1.2$, serum albumin $\geq 3.5 \mathrm{~g} / \mathrm{dl}$ and total serum bilirubin $\leq 1.2 \mathrm{mg} / \mathrm{dl}$.

Also patients were excluded in the case of inadequately controlled diabetes mellitus $\left(\mathrm{HbA}_{1 \mathrm{c}}>9 \%\right)$, total serum bilirubin $>3 \mathrm{mg} / \mathrm{dl}$, HCV-HIV co-infection, $\mathrm{HBV}-\mathrm{HCV}$ co-infection, any cause of chronic liver disease other than hepatitis $\mathrm{C}$, uncontrolled hypothyroidism, hepatocellular carcinoma, except 4 weeks after intervention aiming at cure with no evidence of activity by dynamic imaging (computed tomography [CT] or magnetic resonance imaging [MRI]), extra-hepatic malignancy except after two years of disease-free interval, patients Child's $\mathrm{C}$ cirrhotic and creatinine kinase $>350 \mathrm{u} / \mathrm{l}$.

\section{Treatment intervention}

One hundred twenty-four patients were assessed and only one hundred eligible patients were included in the study and were allocated to either treatment A: drug or treatment B: placebo using the 4-sized block randomization method with possible sequenc- 
es: $\mathrm{ABBA}, \mathrm{BABA}, \mathrm{ABAB}, \mathrm{AABB}, \mathrm{BAAB}$, and BBAA. Random selection among the 6 different blocks was done using a list of random numbers generated by the software SPSS (SPSS Inc., Chicago, IL, USA). Drug group: 50 patients received low dose statin (simvastatin $10 \mathrm{mg}$ ) plus sofosbuvir $400 \mathrm{mg} /$ daclatasvir $60 \mathrm{mg}$ daily (SOF/DAC) for 12 weeks. Placebo group: 50 patients received a starch-based placebo tablet, sofosbuvir $400 \mathrm{mg} /$ daclatasvir $60 \mathrm{mg}$ daily for 12 weeks. SOF/ DAC were obtained from Pharco pharmaceuticals, Egypt. Simvastatin film coated tablets were obtained from Merck Sharp \& Dohme Corp. The placebo tablets were manufactured by Eva Pharma Company, Egypt.

\section{Baseline data}

Demographic data were recorded and included age, gender, body mass index (BMI), hematological tests, liver biochemical profile (serum alanine aminotransferase [ALT] and aspartate aminotransferase [AST]), lipid profile (fasting serum cholesterol, TG, LDL, high-density lipoprotein (HDL) in addition to $\mathrm{HbA}_{1 \mathrm{c}}$, fasting blood glucose, creatinine kinase (CK), thyroid functions (T3 and $\mathrm{T} 4$ ) and $\mathrm{C}$-reactive protein (CRP) at baseline.

\section{End points and follow-up data}

The primary efficacy endpoint was the percentage of patients achieving an sustained virological response at 12 weeks after treatment (SVR12) defined as HCVRNA below the assay's lower limit of detection (LLOD) measured at 12 weeks after the end of treatment. Secondary efficacy endpoints included end of treatment (EOT) virological response, defined as HCV-RNA below the assay's LLOD measured at the EOT. Virological failure included relapse (HCV-RNA $\geq$ LLOD during any post-treatment follow-up visit in patients with HCV-RNA < LLOD at the EOT, virological non-response (HCV-RNA $\geq$ LLOD at EOT).

\section{Safety assessments}

All patients were asked to report any adverse effects (AEs) that may be attributed to the drug during the follow-up period. Safety endpoints included serious AEs, discontinuations due to AEs, deaths, and laboratory abnormalities.

Laboratory tests for assessments of biochemical and hematological parameters, and safety assessments were done as follows: complete blood count (CBC), liver function test, lipid profile and CK every month until the end of therapy; $\mathrm{HbA}_{1 \mathrm{c}}$ and $\mathrm{CRP}$ at the end of therapy; HCV-PCR test at the EOT and 12 weeks after the EOT; fibrosis using FIB-4 12 weeks after the end of therapy.

\section{Statistical analysis}

Data were collected, checked and data entry, processing and statistical analysis was carried out using Med Calc ver. 15.8. Mann-Whitney's $U$-test was used to assess the statistical significance of the difference between two study group means. The $\chi^{2}$ test was used to examine the relationship between two qualitative variables. Repeated measures ANOVA test was used to assess the statistical significance of the difference between more than two (paired) study group means. Friedman's test was used to assess the statistical significance of the difference of a non-parametric variable between more than two (paired) study group means. Univariate and multivariable logistic regression analysis was used to identify baseline factors that were predictors of SVR. $P$-values less than 0.05 was considered to be statistically significant. The current study was a pilot study recruiting $100 \mathrm{HCV}$ patients based on the following calculation: where prior data indicate that the success rate (efficacy) is $90 \%$ [16]. If the true success rate for experimental subjects is $98 \%$, we will need to study 100 experimental subjects to be able to reject the null hypothesis that the success rates for experimental and control subjects are equal with probability (power) $80 \%$. The type I error probability associated with this test of this null hypothesis is 0.05 ; we used uncorrected chi-squared statistics to evaluate this null hypothesis.

\section{Results}

\section{Patients' characteristics}

This prospective study enrolled 100 treatment-naïve chronic hepatitis $\mathrm{C}$ patients: 50 patients received $\mathrm{SOF} /$ DAC treatment in addition to simvastatin (drug group) and 50 control patients treated with SOF/DAC plus a placebo tablet (placebo group). All included patients completed 12 weeks of the protocol regimen with no reported clinically significant side effects, no dose modification needed or withdrawal. All study participants were subjected to 12 weeks of follow-up after cessation of therapy. The baseline demographic data are represented in Table 1 . No significant difference as regards age, weight, height and BMI was found between the two groups $(p>0.05)$. Also, no significant difference was found as regards the baseline laboratory variables and the prevalence of diabetes mellitus (DM) or hypertension $(\mathrm{HTN})$ in the two groups $(p>0.05)$. Comparative study between the 2 groups revealed no 
Table 1. Baseline demographic data of patients in the two groups

\begin{tabular}{lccc}
\hline Variable & $\begin{array}{c}\text { Drug group } \\
(\boldsymbol{n}=50)\end{array}$ & $\begin{array}{c}\text { Placebo group } \\
(\boldsymbol{n}=50)\end{array}$ & P-value \\
\cline { 2 - 3 } & Median (IQR) & $\begin{array}{c}\text { Median (IQR) } \\
\end{array}$ & \\
\hline Age (years) & $59.5(50-65)$ & $58.5(44-65)$ & 0.44 \\
\hline Body mass (kg) & $75.5(68-85)$ & $75(70-85)$ & 0.78 \\
\hline Height $(\mathrm{cm})$ & $170(165-174)$ & $173(165-175)$ & 0.29 \\
\hline BMI (kg/m²) & $2592(24.22-29.41)$ & $25.8(23.5-29.1)$ & 0.72 \\
\hline IQR - interquartile range, BMI - body mass index, statistical test - Mann-Whitney test
\end{tabular}

Table 2. Comparison of treatment responses between drug group and placebo group

\begin{tabular}{lccc}
\hline Parameter & $\begin{array}{c}\text { Drug } \\
\text { group } \\
(n=50)\end{array}$ & $\begin{array}{c}\text { Placebo } \\
\text { group } \\
(n=50)\end{array}$ & $P$-value \\
\hline End of treatment response (ETR) & & & \\
\hline Number (\%) & $50(100)$ & $49(98)$ & 0.317 \\
\hline $95 \% \mathrm{Cl}$ & - & $(97.2-98.4)$ & \\
\hline SVR12 rates & & & \\
\hline SVR12, $n(\%)$ & $49(98)$ & $45(90)$ & 0.207 \\
\hline $95 \% \mathrm{Cl}$ & $(97.8-98.6)$ & $(88.7-90.9)$ & \\
\hline Non-SVR12, $n(\%)$ & $1(2)$ & $5(10)$ & \\
\hline Relapse rates (\%) & & & \\
\hline Relapse (\% of patients with ETR) & $1(2)$ & $4(8.1)$ & 0.246 \\
\hline
\end{tabular}

significant difference as regards baseline Child-Pugh class assessment and fibro-scan staging $(p>0.05)$.

\section{Treatment response}

SVR12 rates were higher among patients in the drug group than among those in the placebo group (98.0\% [95\% CI: 97.8-98.6] vs. 90.0\% [95\% CI 88.790.9], $p=0.207$ ). EOT response was $100 \%$ vs. $98 \%$ in the drug group and placebo group respectively. Primary non-response occurred slightly more often among those who were not treated with statin $(2.0 \%$ vs. $0.0 \%$, $p=0.317$ ) (Table 2).

\section{Follow-up values}

Analysis and comparison of the serial laboratory measurements (baseline, 1st, 2nd, 3rd and 6th months after therapy) of patients in both groups revealed that the average platelets, total leucocyte count (TLC) and neutrophils were increased during serial measurements with a highly significant difference $(p<0.001$ respectively). The average hemoglobin, ALT, AST, fetal bovine serum (FBS), $\mathrm{HbA}_{1 \mathrm{c}}$ and CRP levels were also decreased during serial measurements with a highly significant difference $(p<0.001)$ in both groups.

Regarding lipid profile measurements for patients in the drug group, the average total cholesterol, LDL and TGs levels were initially increased at 1 month, then decreased during serial measurements (especially during 3-month follow-up measurement) with a highly significant difference $(p<0.001)$. On the other hand, the average HDL level was increased during serial measurements with a highly significant difference $(p<0.001)$. However in the placebo group, the average total cholesterol, LDL, TGs and HDL levels of patients were increased during the whole serial measurements with a highly significant difference $(p<0.001)$.

Comparison between the drug group and placebo group HCV patients as regards serial laboratory measurements using repeated measures ANOVA test showed a significant difference between the two groups regarding total cholesterol and LDL levels during serial laboratory measurements with $p<0.003$ and $p<0.039$ respectively.

Also, in the drug group comparing baseline and follow-up fibro-scan measurements revealed a marked increase in stage shift towards class F0 and F1 after therapy, with a significant difference $(p=0.048)$, while in the placebo group comparing baseline and follow-up fibro-scan measurements revealed no significant difference after therapy $(p>0.05)$ (Table 3$)$.

\section{Virological and biochemical response}

Correlation studies between SVR outcome and its relative independent predictors (baseline laboratory

Table 3. Follow-up fibro-scan values for drug group and placebo group

\begin{tabular}{|c|c|c|c|c|c|c|c|}
\hline \multirow[t]{2}{*}{ Variable } & & \multicolumn{3}{|c|}{ Drug group $(n=50)$} & \multicolumn{3}{|c|}{ Placebo group $(n=50)$} \\
\hline & & Baseline $n(\%)$ & $\begin{array}{c}\text { Follow-up (12 weeks) } \\
n(\%)\end{array}$ & $p$-value & Baseline $n(\%)$ & $\begin{array}{c}\text { Follow-up (12 weeks) } \\
n(\%)\end{array}$ & $p$-value \\
\hline \multirow[t]{5}{*}{ Fibro-scan } & F0 & $9(18)$ & $12(24)$ & 0.048 & $12(24)$ & $4(8)$ & 0.479 \\
\hline & $\mathrm{F} 1$ & $8(16)$ & $13(26)$ & & $7(14)$ & $10(20)$ & \\
\hline & $\mathrm{F} 2$ & $6(12)$ & $7(14)$ & & $4(8)$ & $8(16)$ & \\
\hline & $\mathrm{F} 3$ & $10(20)$ & $10(20)$ & & $13(26)$ & $8(16)$ & \\
\hline & $\mathrm{F} 4$ & $17(34)$ & $8(16)$ & & $14(28)$ & $20(40)$ & \\
\hline
\end{tabular}


variables) were conducted. Logistic regression analysis showed that the increase in baseline CRP and the decrease in baseline hemoglobin level, and the non-statin usage had an independent effect on increasing the probability of SVR failure in both groups, with a statistically significant difference $(p=0.033, p=0.0028$, $p=0.027$ respectively) (Table 4$)$.

\section{Discussion}

The main target for HCV treatment is to eradicate hepatitis C virus RNA. This can be predicted by the attainment of SVR, which is associated with a $99 \%$ chance of being HCV RNA negative during longterm follow-up [17], as well as a decrease in all HCVrelated mortality, liver-related death, the need for liver transplantation, hepatocellular carcinoma rates, and liver-related complications [18]. It is well known that the HCV replication process can be disrupted by treatment with inhibitors of 3-hydroxy-3-methyglutaryl CoA (HMGCoA) reductase such as statins [19]. Thus statins may represent an interesting adjuvant to the standard of care therapy to improve the sustained virological response in HCV patients receiving combination antiviral therapy [20].

In the current study, both groups of patients showed significant reduction in ALT and AST at the end of treatment compared to the baseline values $(p<0.0001)$; the results were in accordance with those reported by Mohamed et al. [21]. Regarding lipid profile measurements, in the drug group the average total cholesterol, LDL and TGs levels were initially increased at 1 month, then decreased during serial measurements (especially during 3-month follow-up measurement) with a highly significant difference $(p<0.001)$, while in the placebo group, the average total cholesterol, LDL, TGs and HDL levels continued to increase during serial measurements with a highly significant difference $(p<0.001$ respectively).

In agreement with these results Mohamed et al. also reported that $\mathrm{HCV}$-statin group patients had additional statistically significant reduction of their total cholesterol, triglycerides and HDL at the end of treatment compared to baseline $(p=0.0001)$ while such a significant reduction was not observed in the HCVnon statin group [21].

Comparisons of serum lipid profiles after treatment showed a similar increase in TC and LDL-C levels between the DAC + ASV-SVR and SOF + LDV-SVR groups [5]. Results reported by Jain and his colleagues showed that total cholesterol levels $(166.9 \pm 23.8$ to $192.4 \pm 34.5 \mathrm{mg} / \mathrm{dl}, p<0.0001)$ and LDL-cholesterol levels $(100.9 \pm 22.8$ to $121.6 \pm 37.2, p<0.0001)$ were
Table 4. Multivariate logistic regression model for the pretreatment variables associated with SVR

\begin{tabular}{lccc}
\hline Independent variables & Coefficient & Std. error & $P$-value \\
\hline $\mathrm{Hgb}(\mathrm{g} / \mathrm{dl})$ & -0.87887 & 0.28689 & $0.0022^{*}$ \\
\hline $\mathrm{CRP}(\mathrm{mg} / \mathrm{dl})$ & 0.038907 & 0.018294 & $0.033^{*}$ \\
\hline Drug group & -3.41681 & 1.54947 & $0.027^{*}$ \\
\hline
\end{tabular}

Drug group - "Statin-SOF/DAC", Standard error - It is the standard deviation of the sampling distribution of the mean.

*statistically significant $p>0.05$

elevated after the treatment [10]. So according to the results of the previous studies SOF/DAC was chosen in our study to evaluate the clinical benefits that may be seen by addition of simvastatin to that regimen.

In this study, both groups of patients showed significant reduction in $\mathrm{HbA}_{1 \mathrm{c}}$ at the end of treatment compared to baseline values, $p<0.0001$, which shows that successful treatment may improve glycemic control. Moreover, in the drug group patients who received low dose simvastatin CRP values were significantly reduced after 3 months of treatment while in the placebo group they were significantly increased. These results may suggest additional benefits of statin as an anti-inflammatory agent and also its role in reducing metabolic syndrome. These results are in agreement with Butt and his colleagues, who were able to show in a multivariate model that the antifibrotic benefits observed in statin users remained significant even after adjustment not only for SVR, but also for BMI and the presence of underlying diabetes, suggesting that the protective effect of statins extends beyond modification of metabolic syndrome and obesity-related inflammation [11].

Assessment of the degree of fibrosis revealed a significant increase in percentages of patients with $\mathrm{F} 0$ and F1 stages in the drug group compared to the placebo group during follow-up assessment $(p=0.0435)$. In agreement with the above results, a post-hoc analysis of a large prospective human trial of patients with advanced $\mathrm{CHC}$ followed with serial liver biopsies, it was demonstrated that statin use was associated with significantly reduced fibrosis scores [11].

Regarding treatment response, this study showed an increase in treatment relapse rates in the placebo group $(8.1 \%)$ compared to the drug group (only $2 \%$ ); also EOT response and SVR12 rate were higher in the drug group versus the placebo group but not reaching a statistically significant difference $(p>0.05)$. Zhu et al. reported that several randomized controlled trials have demonstrated that statins increase SVR rates when combined with PEG-interferon and ribavirin in genotype 1 infection [22]. Moreover Moriguchi et al. published two reports in 2010 investigating the anti- 
viral efficacy of pitavastatin against $\mathrm{HCV}$ infection in vitro and demonstrated the antiviral efficacy and safety of pitavastatin against HCV infection [23]. These effects, the antiviral efficacy and safety of pitavastatin against $\mathrm{HCV}$ infection were further confirmed in two clinical studies [24]. Also, Butt et al. found that statin use was significantly associated with decreased progression of fibrosis independent of having attained an SVR and demonstrated the effects of statins on all three critical clinical outcomes of HCV, including fibrosis progression, SVR, and HCC development, in an unselected group of patients with various stages and genotypes of HCV [11]. In contrast to our results Abd-Eldaem et al. showed that addition of fluvastatin to pegylated interferon and ribavirin significantly increased the sustained virological response from $55 \%$ to $62.5 \%(p<0.01)$ and significantly decreased viral load in relapse patients $(p<0.001)$. No significant differences and correlations were found between serum levels of LDL-cholesterol and viral load before and after treatment in both groups [25].

Correlation studies between SVR outcome and its relative independent predictors (baseline laboratory variables) were conducted with logistic regression analysis and showed that only high baseline CRP, low baseline Hgb level and no statin usage had an independent effect on increasing the probability of SVR failure in both groups with a statistically significant difference ( $p=0.033, p=0.0028, p=0.027$ respectively). In agreement with the results of this study Mohamed et al. also reported that statin use was much more significantly associated with SVR, with a $p$-value of 0.003 [21].

Moreover, Butt et al. showed in a multivariate Cox's regression analysis that treatment with statins was associated with a higher likelihood of achieving SVR (odds ratio $[\mathrm{OR}]=1.44 ; 95 \% \mathrm{CI}=1.29-1.61$ ) $[11]$.

Regarding safety data of both regimens in HCV patients, all patients in both groups continued the whole study period with no withdrawal due to treatment schedule side effects, confirming data on the safe use of statin in HCV infected patients.

In conclusion, statins could have an irreplaceable role in the successful treatment and prognosis of $\mathrm{CHC}$ patients receiving sofosbuvir/daclatasvir.

\section{Disclosure}

The authors report no conflict of interest.

\section{References}

1. Simon TG, Butt AA. Lipid dysregulation in hepatitis $\mathrm{C}$ virus, and impact of statin therapy upon clinical outcomes. World J Gastroenterol 2015; 21: 8293-8303.
2. Gomaa A, Allam N, Elsharkawy A, et al. Hepatitis C infection in Egypt: prevalence, impact and management strategies. Hepat Med 2017; 9: 17-25.

3. Pedersen MR, Patel A, Backstedt D, et al. Genotype specific peripheral lipid profile changes with hepatitis $C$ therapy. World J Gastroenterol 2016; 22: 10226-10231.

4. Gitto S, Cicero AFG, Loggi E, et al. Worsening of serum lipid profile after direct acting antiviral treatment. Ann Hepatol 2018; 17: 64-75.

5. Endo D, Satoh K, Shimada N, et al. Impact of interferon-free antivirus therapy on lipid profiles in patients with chronic hepatitis C genotype 1b. World J Gastroenterol 2017; 23: 2355-2364.

6. Lange CM, von Wagner M, Bojunga J, et al. Serum lipids in European chronic HCV genotype 1 patients during and after treatment with pegylated interferon-alpha-2a and ribavirin. Eur J Gastroenterol Hepatol 2010; 22: 1303-1307.

7. Hsu YC, Ho HJ, Huang YT, et al. Association between antiviral treatment and extrahepatic outcomes in patients with hepatitis C virus infection. Gut 2015; 64: 495-503.

8. Sun HY, Cheng PN, Tseng CY, et al. Favouring modulation of circulating lipoproteins and lipid loading capacity by direct antiviral agents grazoprevir/elbasvir or ledipasvir/sofosbuvir treatment against chronic HCV infection. Gut 2018; 67: 13421350.

9. Meissner EG, Lee YJ, Osinusi A, et al. Effect of sofosbuvir and ribavirin treatment on peripheral and hepatic lipid metabolism in chronic hepatitis $\mathrm{C}$ virus, genotype 1-infected patients. Hepatology 2015; 61: 790-801.

10. Jain A, Kalra BS, Srivastava S, Chawla S. Effect of sofosbuvir and daclatasvir on lipid profile, glycemic control and quality of life index in chronic hepatitis $\mathrm{C}$, genotype 3 patients. Indian J Gastroenterol 2019; 38: 39-43.

11. Butt AA, Yan P, Bonilla H, et al. Effect of addition of statins to antiviral therapy in hepatitis $C$ virus-infected persons: Results from ERCHIVES. Hepatology 2015; 62: 365-374.

12. Dimitroulakos J, Lorimer IA, Goss G. Strategies to enhance epidermal growth factor inhibition: targeting the mevalonate pathway. Clin Cancer Res 2006; 12: 4426s-4431s.

13. Zhao TT, Le Francois BG, Goss G, et al. Lovastatin inhibits EGFR dimerization and AKT activation in squamous cell carcinoma cells: potential regulation by targeting rho proteins. Oncogene 2010; 29: 4682-4692.

14. Delang L, Paeshuyse J, Vliegen I, et al. Statins potentiate the in vitro anti-hepatitis $C$ virus activity of selective hepatitis $C$ virus inhibitors and delay or prevent resistance development. Hepatology 2009; 50: 6-16.

15. Kishta SS, Kishta SA, El-Shenawy R. Statin (3-hydroxy-3 methylglutaryl-coenzyme A reductase inhibitor)-based therapy for hepatitis $\mathrm{C}$ virus $(\mathrm{HCV})$ infection-related diseases in the era of direct-acting antiviral agents. F1000 Research 2017; 5: 223.

16. Fontaine H, Hezode C, Zoulim F, et al. Efficacy of the oral sofosbuvir-based combinations in HCV genotype 4-monoinfected patients from the French observational cohort ANRS CO22 Hepather. In: Proceedings of the 50th Annual Meeting of European Association for the Study of the Liver. Vienna, Austria, April 2015.

17. Swain MG, Lai MY, Shiffman ML, et al. A sustained virologic response is durable in patients with chronic hepatitis $\mathrm{C}$ treated with peginterferon alfa-2a and ribavirin. Gastroenterology 2010; 139: 1593-1601.

18. Backus LI, Boothroyd DB, Phillips BR, et al. A sustained virologic response reduces risk of all-cause mortality in patients with hepatitis C. Clin Gastroenterol Hepatol 2011; 9: 509-516.e501. 
19. Kapadia SB, Chisari FV. Hepatitis C virus RNA replication is regulated by host geranylgeranylation and fatty acids. Proc Natl Acad Sci U S A 2005; 102: 2561-2566.

20. Rao GA, Pandya PK. Statin therapy improves sustained virologic response among diabetic patients with chronic hepatitis C. Gastroenterology 2011; 140: 144-152.

21. Mohamed AA, El-Halawany F, El-Nabarawy N, et al. Statins added to chronic hepatitis $\mathrm{C}$ treatment: is it beneficial? J Hepatol Gastroint Dis 2016; 2: 117

22. Zhu Q, Li N, Han Q, et al. Statin therapy improves response to interferon alfa and ribavirin in chronic hepatitis $\mathrm{C}$ : a systematic review and meta-analysis. Antiviral Res 2013; 98: 373-379.

23. Moriguchi H, Chung RT, Sato C. New translational research on novel drugs for hepatitis $\mathrm{C}$ virus $1 \mathrm{~b}$ infection by using a replicon system and human induced pluripotent stem cells. Hepatology 2010; 51: 344-345.

24. Kohjima M, Enjoji M, Yoshimoto T, et al. Add-on therapy of pitavastatin and eicosapentaenoic acid improves outcome of peginterferon plus ribavirin treatment for chronic hepatitis C. J Med Virol 2013; 85: 250-260.

25. Abd-Eldaem AA, Azmy MK, Ahmad YK, et al. Role of statins in the treatment of chronic hepatitis $\mathrm{C}$ virus infection. J Egypt Soc Parasitol 2012; 42: 535-540. 